\title{
The Evolution of Sports: Exploring Parental Interest in Watching Sports
}

\author{
Menelaos Apostolou ${ }^{1}$ - Marianna Zacharia ${ }^{1}$
}

Published online: 23 April 2015

(C) Springer International Publishing 2015

\begin{abstract}
Across different cultures and different times, people maintain a strong interest in watching other people, and especially, men playing sports. The present paper employs an evolutionary perspective in order to understand how selection forces have shaped this interest. In particular, it is hypothesized that individuals, and particularly men, have evolved an interest to watch other men competing in athletic contest in order to be able to identify useful allies and spouses for their daughters. On this basis, four predictions are derived: people are more interested in watching men than women competing; men are more interested in watching men than women competing; men with daughters are more interested in watching sports, and, finally, men with daughters are more interested in watching men than women competing. A study of 514 parents finds evidence consistent with all four predictions.
\end{abstract}

Keywords The evolution of sports · Male-male competition . Parental choice

\section{Introduction}

People watching athletic competitions is a pattern which appears to be present in almost all modern and ancient cultures (Deaner and Smith 2013; Guttmann 1986, 2004; Kyle 2006). Watching a sport event requires the commitment of resources, including time and money (i.e., to purchase a ticket) with the only apparent benefit of this being entertainment. In addition,

Menelaos Apostolou

m.apostolou@gmail.com

1 University of Nicosia, 46 Makedonitissas Ave., 1700 Nicosia, Cyprus it appears that most sports involve predominantly men competing in front of a male audience. These observations raise the question of whether, and if so why, selection forces have shaped the male, and not the female mind, to be interested in doing something that incurs direct costs without apparent survival benefits. Two contemporary theories argue that these behaviors have been favored by sexual selection forces (Apostolou 2014c; Lombardo 2012). Based on this evolutionary framework, previous research has derived and tested several hypotheses (Apostolou et al. 2014). The purpose of the present research is to advance this work by testing additional hypotheses, and by replicating its findings in a larger sample.

\section{Why are Men Interested in Watching Other Men Competing?}

Participating in sports and watching sports requires devoting resources such as energy, time, and money. In the evolutionary perspective, any predispositions for engaging in behaviors which are costly without providing any survival or reproductive benefits would be eliminated (Tooby and DeVore 1987). Apparently, selection forces have not eliminated the inclination to do and watch sports, which suggests that these predispositions confer, or would have conferred to our ancestors, survival, and/or reproductive benefits. Most scholars support the latter that is, they argue that sports were favored by selection forces because of the reproductive advantages they conferred.

In particular, it has been argued that sports are likely to function as culturally invented courtship rituals that reliably advertise unobserved athletic abilities to the opposite sex (Miller 2000; Zahavi and Zahavi 1997). More specifically, women divert more parental investment to their children (e.g., 9 months of gestation and breastfeeding), and thus, they become the scarce reproductive resource to which men strive 
to gain access (Trivers 1972). This places women in a position where they can exercise choice, and when they do, they tend to prefer men with qualities which are most beneficial for them (Buss 2003).

Since good athletic ability correlates with several factors (e.g., fighting ability, stamina, strength) that women are interested in and looking for in a mate, it pays for them to prefer men with superior athletic abilities, and it pays for men to demonstrate these abilities (Miller 2000; Zahavi and Zahavi 1997). Men can do so by participating in athletic competitions in which they can compete in a standardized fashion, signaling their aptitude and simultaneously protecting themselves from cheaters (Zahavi and Zahavi 1997). This is possible because athletic contests involve considerable physical strain; thus, men with limited physical capacities will be unable to compete, enabling the ones with superior physical capacities to stand out as winners. Consequently, cheating becomes more difficult and thus less frequent. On this basis, it has been proposed that intersexual competition driven by female choice gave rise to athletic contests, which evolved to enable men to reliably and accurately signal their unobserved physical abilities to women (De Block and Dewitte 2009; Miller 2000; Zahavi and Zahavi 1997).

Men can also gain mating access to the opposite sex by excluding other competitors through force (Andersson 1994; Puts 2010). One way for men to do so is to fight other men and monopolize access to women. They can succeed in this by establishing coalitions with other men. In particular, men who pick good allies have a selective advantage over those who do not, which means that selection pressures are exercised on men to be able to distinguish between potential allies and enemies. Abilities such as strength and speed determine whether a man can be a useful ally. On this basis, Lombardo (2012) argued that intrasexual selection has shaped men to be interested in participating in sports so as to be chosen as allies, and to watch sports so as to be able to determine who can be a valuable ally.

Men can also gain access to the reproductive capacity of women by being selected as sons-in-law. More specifically, conflicting interests over mating induce parents to control the mating decisions of their children and particularly of their daughters, who constitute the scarce reproductive resource (Apostolou 2014b). In a pre-industrial context, and most likely during the greatest part of human evolution, arranged marriage is the prevalent mode of long-term mating, where parents choose spouses for their children. Also, because fathers are physically stronger, control more wealth, and potentially can extract more benefits from arranging a marriage than mothers, it is usually the case that fathers control the mate choices of their daughters (Apostolou 2014a, b).

Abilities such as endurance, strength, and speed determine whether a man can be a valuable son-in-law. On this basis, it has been argued that intersexual selection has also contributed in shaping men to be interested in participating in sports in order to be chosen as sons-in-law and to watch sports so as to be able to distinguish between prospective sons-in-law with desirable qualities (Apostolou et al. 2014).

Empirical evidence favors the male-male competition and the parental choice hypotheses. In particular, the female choice hypothesis proposes that men would engage in athletic competitions in order to signal their unobserved abilities to women in the audience. Yet, women are usually not in the audience, which is dominated by men (Apostolou 2014c; Guttmann 1986, 2004). This suggests that the interest to do and watch athletic competitions have evolved predominantly to enable reliable communication between men.

\section{Hypotheses on the Interest to Watch Sports}

The above theoretical framework can be employed for deriving specific hypotheses on the willingness to watch sports. To begin with, it is men who are predominantly selected as allies, and it is men who are predominantly selected as in-laws. Accordingly, the selection pressure would be stronger on individuals to be interested in the athletic performance of men than of women. Thus, it can be predicted that individuals would be more interested in watching men than women competing (Apostolou et al. 2014). Furthermore, as it is men who predominantly look for male allies and sons-in-law, these selection pressures would be stronger on men than on women. In turn, this predicts an interaction effect, with men being more interested than women in watching men than women competing (Apostolou et al. 2014). One study found evidence in favor of both hypotheses (Apostolou et al. 2014), and the present research aims to replicate these findings in a larger sample.

Moreover, in this theoretical framework, there is parental interest in the athletic performance of men who can become sons-in-law. This indicates that men who have female children would be more interested than men (and women) who have male children in watching sports. That is, an interaction effect is expected to arise between the sex of the child that parents have and the sex of the parent. Furthermore, as it is predominantly men who are sought as allies and sons-in-law, we would predict that fathers with female children would be more interested than parents with male children in watching men competing than women competing. That is, a three-way interaction between the sex of the parent, the sex of the child, and the sex of the athlete is expected to arise. The present research aims to test these hypotheses. The parental interest in watching sports was not examined by Apostolou et al. 2014 study, and this is a novel contribution of this research. 


\section{Methods}

\section{Participants}

Four research assistants were employed for the purposes of this study. They recruited individuals who volunteered to participate in a research on sports. To qualify for participation, an individual had to be an adult and have at least one child. A snowball sampling technique was used where the research assistants recruited first several participants and then obtained references for other participants who may be interested in participating in the study. Participants completed the study at their homes. The study took place in the Republic of Cyprus, and the participants came predominantly from the two biggest cities of the country, namely Nicosia and Limassol. The data collection process lasted approximately 2 months. The participants were initially asked to sign a consent form, and then they were given the survey. Upon completion, the participants put the questionnaire in an unmarked envelope and sealed it.

In this study, 514 Greek-Cypriots took part (252 men, 262 women). The mean age of women was $41.9(S D=9)$, and the mean age of men was $45.2(S D=9.5)$. Participants had a mean of $1.4(S D=0.6)$ male children and a mean of $1.5(S D=0.7)$ female children. The mean age of the oldest female child was 16.6 $(S D=9.5)$, and the mean age of the oldest male child was $16.7(S D=9.6)$. Moreover, $83.9 \%$ of the participants were married, $9.3 \%$ were divorced, $3.9 \%$ were in a relationship, $1.8 \%$ were single, and $1.2 \%$ were widowed.

\section{Materials}

The survey consisted of two parts. In the first part, participants were given the following scenario: "You are visiting a different country and you find out that in the next few days several athletic events will take place. Indicate how willing you would be to physically attend or watch these events on television." This scenario was chosen because certain sports (e.g., ski) are not widely practiced in the country where this study took place (i.e., Cyprus). The instrument used was the one originally developed by Apostolou et al. (2014) and contained 19 different sports. In addition, the method we employed is identical with the one by Apostolou et al. (2014), with the difference being that in this study we have employed only parents and we have measured demographic characteristics associated with this (e.g., number of children).

These sports were presented to participants in two versions: in one version, men were competing (e.g., men's basketball), and in the other version, women were competing (e.g., women's basketball). Overall, participants had to rate 38 athletic events, and their answers were recorded in a five-point Likert scale (one-very unwilling, five-very willing). In the second part, demographic information was collected (i.e., sex, age, marital status, number of male children, number of female children, age of oldest male child, age of oldest female child).

\section{Results}

In this study, the interest in watching sports spans across 19 different sports; accordingly, for the purposes of this research, a doubly multivariate analysis was conducted. The simplest way to view this statistical procedure is to be like a MANOVA which allows one or more of the independent variables to be within-participants (in this case, the sex of the athletes competing as participants gave rating for both the male and the female version of the sport) (see Tabachnick and Fidell 2012). Accordingly, all the different sports entered as the dependent variables, and the type of sport (men competing, women competing), the participants' sex, and the type of children (male, female, both) entered as independent variables. Finally, participants' age entered as a covariate.

The results are presented in Table 1 where the sports were placed in a hierarchical order, with those receiving the highest score at the top. In order to do so, we estimated the mean for each sport by averaging the scores given for the male and the female version of the sport. We can see that a significant main effect of the type of sport is found for the majority of sports, with participants indicating that they are more interested in watching men than women competing. There are two exceptions, namely gymnastics and aerobics, where the difference is to the opposite direction. It can also be seen that for the majority of sports, there is a significant interaction between the type of sport and the participants' sex: when participants shift their viewing preference from a female sport to a male sport, men's interest increases more than women's. This interaction effect can be seen in Fig. 1.

From Table 1, we can also see that there is a significant interaction between the sex of the parent and the sex of the child, with parents having female children being more interested in watching sports than parents who have male children or children of both sexes. This effect is found for several sports, and it has to be said that for several other sports, the interaction effect approached significance without however passing it.

Also, we can see that there is a significant interaction between the type of children, the sex of the parent, and the type of sport, with men with female children preferring more than everybody else to watch men competing. As in the case above, this effect came significant for some sports; yet, for several other sports, it did not pass the significance level. Still, for several sports, the $p$ value was close to the significance level with the means being to the predicted direction. Note also that for aerobics, the three-way interaction came significant, but the means were not to the predicted direction, with mothers of 


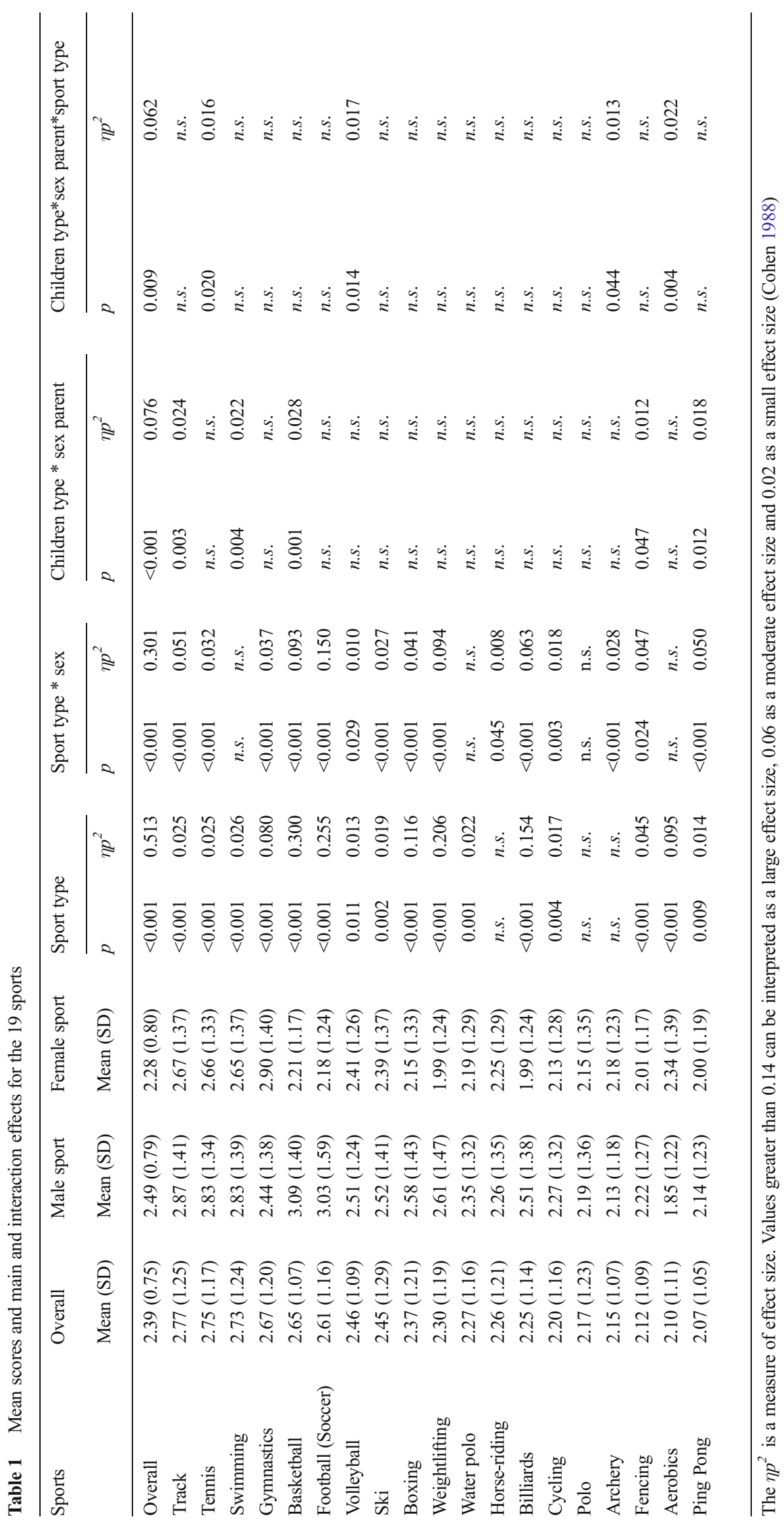




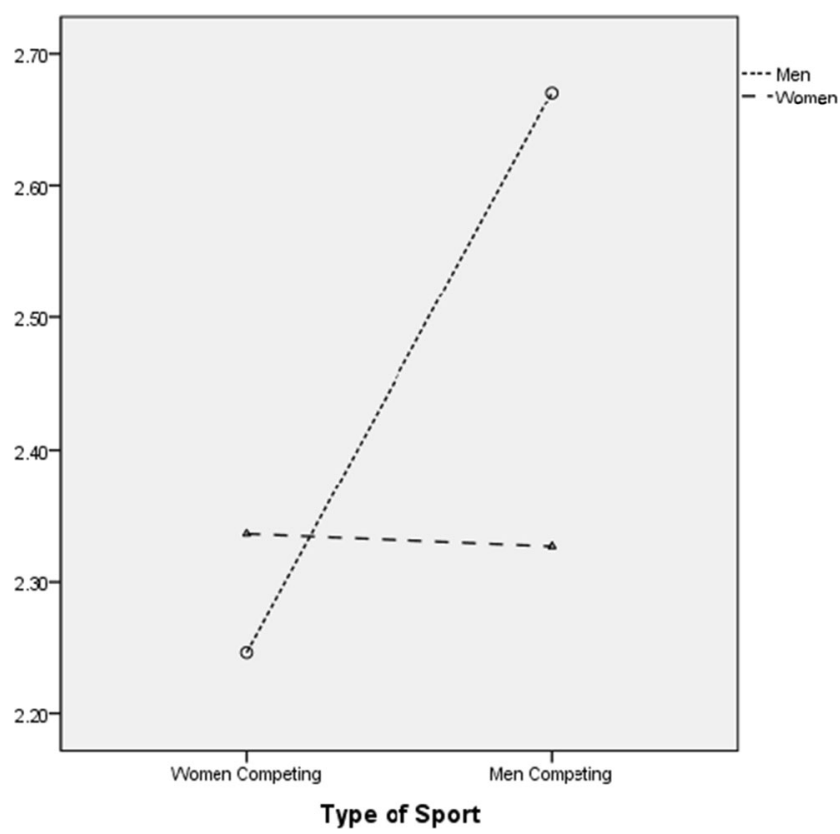

Fig. 1 Interaction effect between participants' sex and the type of sport. We can see that men and women have a similar interest in watching women competing, but when we shift to men competing, men's interests in watching increases considerably, but women's interest remains relatively unaffected

both male and female children preferring to watch the female version of the sport, giving the highest score.

These interaction effects are depicted in Figs. 2, 3, and 4. Figure 2 depicts the interaction effect between the sex of the parent and the sex of the child on the overall interest to watch sports. We can see that men with female children have the highest mean (note, however, that it is not significantly

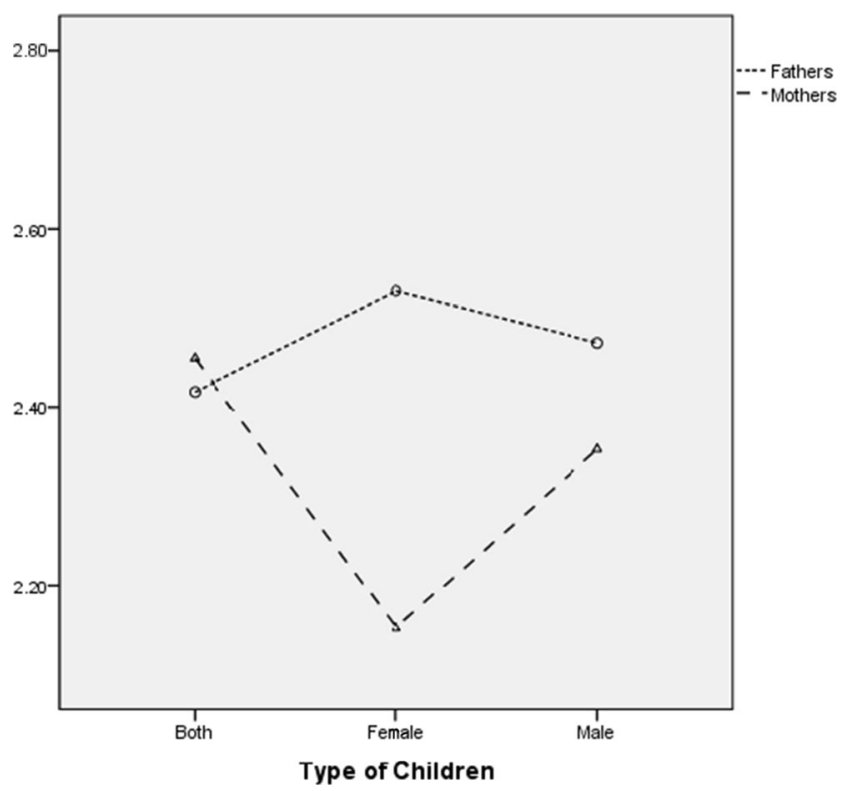

Fig. 2 Interaction effect between the sex of the parent and the type of children for the interest to watch sports. We can see that fathers who have only female children exhibit the strongest interest in watching sports

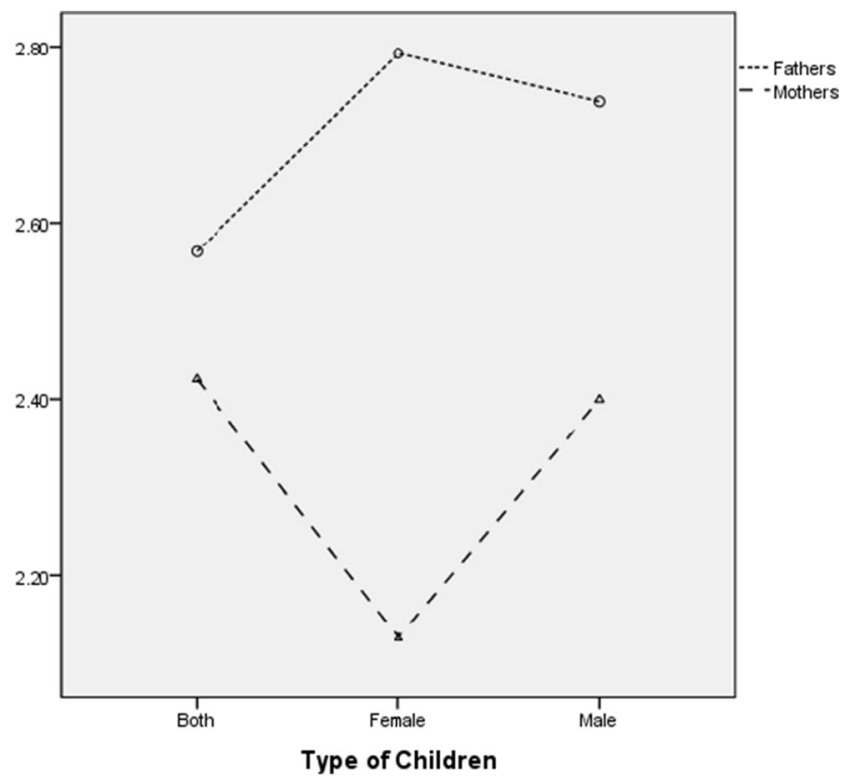

Fig. 3 Interaction effect between the sex of the parent and the type of children for the interest to watch male sports. We can see that fathers who have only female children exhibit the strongest interest in watching sports

different from the mean of men who have only sons), while the biggest difference in the interest to watch sports is between fathers and mothers who have only female children, with the former exhibiting a much stronger interest than the latter. Figures 3 and 4 decompose this effect for the interest to watch male and female sports. In particular, Fig. 3 depicts the interaction effect for the interest to watch male sports, and Fig. 4 depicts the interaction effect for the interest to watch female sports. We can observe that when parents have only daughters,

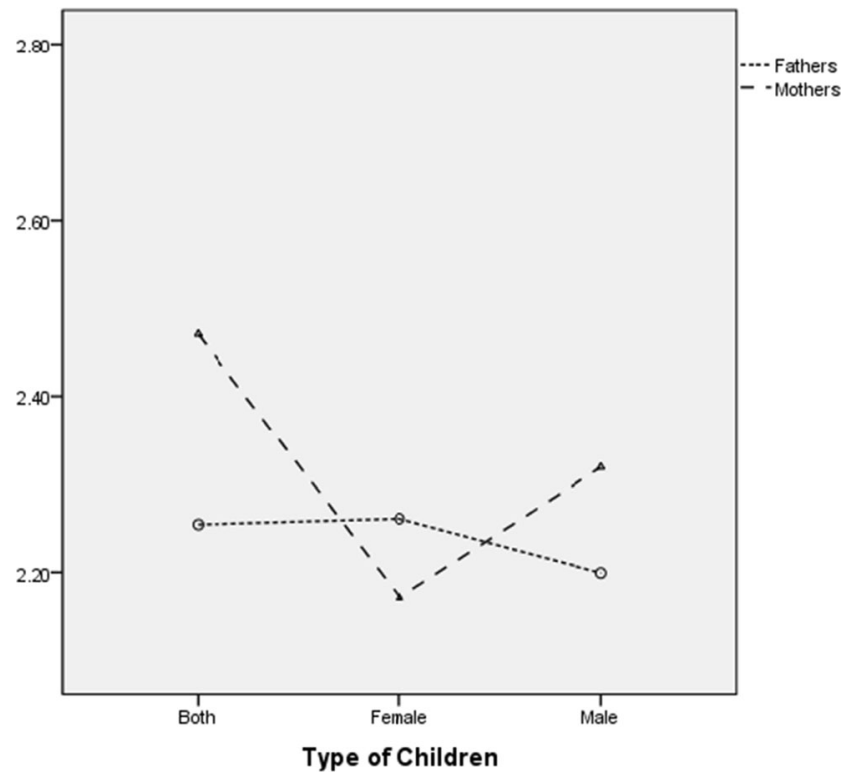

Fig. 4 Interaction effect between the sex of the parent and the type of children for the interest to watch female sports. As opposed to male sports, here, fathers who have daughters do not exhibit the strongest interest to watch 
the difference between fathers and mothers is much more pronounced in the case of male sports than in the case of female sports, suggesting a three-way interaction between the sex of the parent, the sex of the child, and the sex of the athlete.

Note also that the results indicate a significant main effect of $\operatorname{sex}\left[F(19,471)=12.18, p<0.001, \eta p^{2}=0.330\right]$ with men exhibiting a stronger interest to watch sports than women. Note further that the age variable did not come significant.

\section{Discussion}

The results of this research confirm previous findings that both men and women are more interested in watching men than women competing, while men are more interested than women in watching other men competing. In addition, fathers who have daughters are more interested in watching sports than parents who have sons or children of both sexes. Also, fathers who have daughters are more interested in watching male sports. When examining individual sports, these two interaction effects were found for a minority of sports; yet, for many of the non-significant results, the means were to the predicted direction and the $p$ values close to significance, suggesting that a future study based on a larger sample may produce more significant results.

These two interaction effects come predominantly from the mothers interests changing substantially more than the fathers' interests when the sex of the child changes. For instance, when parents have only female children, the father's interest in watching male sports is considerably more than the mother's; however, when we move to the condition of having only male children, the mother's interest increases considerably while the father' $s$ interests decreases marginally. Our theoretical framework predicts that fathers with female children will maintain a higher interest in watching male sports than mothers, which is the case, but it also suggested that the fathers' interest in watching male sports would decline if they have only male children, while the mothers' interest would remain relatively unaffected, which does not appear to be the case.

One possible explanation for this is that in the case where parents have male children, they are concerned about how their sons are doing in relation to other possible competitors. In particular, the fitness of their sons, at least in a pre-industrial context, depends on how they fare in relation to other men in terms of their athletic abilities. Thus, both mothers and fathers would maintain an interest in assessing how their sons are doing in relation to other people's sons. On the other hand, when parents have only daughters, fathers would have a strong interest in watching other men competing, looking for desirable sons-in-laws and allies, but mothers have a lower interest in doing so, as historically, they had less control over mate choice than fathers, and did not form coalitions to fight other groups. In this respect, the difference in the levels of interest to watch male sports found in the case of daughters is likely to reflect an evolved interest to seek useful in-laws and allies, while, in the case of having only sons, the converging interest of parents coming predominantly from the increase in the mothers' interests, is likely to reflect an evolved interest in the success of the male offspring. More theoretical and empirical work is required in order to understand better the observed interaction effects.

It has been argued that the men's interest in watching sports evolved in order to promote the selection of beneficial allies and in-laws. To this, it should also be added that by watching sports, men can determine who is a dangerous opponent, and use this information to avoid getting into conflict with such individuals (Lombardo 2012). Moreover, by watching other men competing, individuals can also benefit from learning competitive techniques, and how to overcome situations that they might encounter while they are competing.

It has to be said at this point, that this paper does not suggest that women do not have an interest in watching sports in general or male sports in particular. The results clearly indicate that women have an interest in watching male and female sport, but when it comes to male sports, their interest is weaker than the men's interest. Furthermore, the hypothesized patterns, such as men being more interested than women in watching other men competing apply to the vast majority of sports examined, but there are exceptions, such as gymnastics, where different patterns are found (i.e., men and women prefer to watch more women than men competing). One possible explanation is that gymnastics is an esthetic sport, resulting into women being superior to men at this on average. Individuals would then prefer to watch women rather than men competing, because in this case, the spectacle would be superior.

Parents only look for long-term partners for their children. Control of resources and a willingness to invest these resources in partners and their families need to be important characteristics, which are of interest to parents in a prospective in-law (see Apostolou 2014b). Such traits cannot be assessed through performance in an athletic contest. Athletic contests can be used to assess predominantly physical abilities, and parents need to resort to other means in order to examine wealth and willingness to provide resources. For instance, in many societies, a man has to pay a considerable amount of wealth, called bridewealth, to his prospective in-laws for the marriage to proceed. By demanding bridewealth, parents can assess the wealth of a prospective in-law and his capacity to devote these resources to his newly established family (Apostolou 2014b). Overall, sports can serve parents in distinguishing specific abilities in a prospective son-in-law, but they cannot serve them in assessing the full range of desirable abilities and traits.

Furthermore, in ancestral human societies, watching sports would have been a local activity watched in person. Today, 
people watch sporting events on television where players from around the world compete against each other, and they are unlikely to ever meet these individuals. This suggests that people would also maintain an interest in watching local athletic contests, even if the spectacle is of inferior quality, because this information is more relevant to them. This can explain, for instance, why in soccer people are strongly interested in watching teams competing in the second or third division although the spectacle is usually inferior to the one in the first division: teams in these divisions usually come from smaller cities or villages, and the spectators are likely to come across or know those who compete.

The observed patterns are unlikely to reflect only evolved predisposition effects. For instance, men are physically stronger, more aggressive, and have a higher stamina than women (Lassek and Gaulin 2009; Seiler et al. 2007), which suggests that in male sports, the spectacle is likely to be more impressive than in female sports, generating a higher interest in watching men than women competing. Nevertheless, such effects are not external to the evolutionary framework, as sexual selection forces are predominantly the reasons why men differ from women in these dimensions.

Future research may attempt to replicate the findings of this study using an experimental methodology. In particular, researchers may provide participants access to a TV set where they have the option to watch several different sports coming in male and female versions, and investigate whether individuals would spend more time watching the male or the female version of the sport. Similarly, a longitudinal study can examine whether having children, especially daughters, increases individuals' interest, especially men's, in watching sports, particularly sports where men are competing.

In this study, the significant main and interaction effects as well as the effect sizes differ across sports. One possible reason behind this variation is likely to be that sports differ in their capacity to signal information about the qualities which are of interest to the observers. Accordingly, future research needs to attempt to understand this variation, starting by identifying the specific qualities that individuals are interested in observing and proceed to assessing different sports on their capacity to signal these qualities.

This study is based on self-report evidence, which is a limitation since individuals may behave differently in actual circumstances than they indicate here. Also, this study is limited to a single culture and future studies need to replicate its findings in different cultural settings. Last but not least, the participants of this study had to be parents, which mean that their age and perhaps other demographic characteristics are not representative of the adult population.

Costly behaviors with no apparent survival benefits are likely to reflect the expression of adaptions which have evolved to serve reproductive purposes. Understanding these behaviors requires that their study is placed in an evolutionary framework, a framework which indicates that these have been shaped in an ancestral context which is different from our own. More specifically, in post-industrial societies, we observe a strong interest in playing and watching sports where men compete before an audience of other men. The evolved nature of this behavior cannot be readily understood in reference to this context where individual mate choice and especially female choice dominate. The evolved nature of this behavior makes much more sense in reference to a context where marriages are arranged by male parents and where men form coalitions to fight other men and monopolize access to women.

This can go the other way around as well: the interest of men to watch other men competing and the interest of fathers who have daughters to watch other men competing can be interpreted as evidence that during human evolutionary time male-male competition and male parental choice had been strong selection forces. Replication of the findings of this study to different cultural contexts will strengthen this argument as it will indicate that these findings reflect evolved predispositions rather than cultural effects.

Overall, the current study offers evidence consistent with the predictions of the evolutionary framework which enables us to understand the interest in watching sports, but more work and replication is necessary if more definite conclusions are to be reached.

Acknowledgments The authors would like to thank two anonymous reviewers for their feedback which enabled the improvement of this work.

\section{References}

Andersson, M. (1994). Sexual selection. Princeton: Princeton University Press.

Apostolou, M. (2014a). Sexual selection under parental choice: a revision to the model. Theory in Biosciences, 133, 111-115.

Apostolou, M. (2014b). Sexual selection under parental choice: the evolution of human mating behavior. Hove: Psychology Press.

Apostolou, M. (2014c). The athlete and the spectator inside the man: A cross-cultural investigation of the evolutionary origins of athletic behavior. Cross-Cultural Research. Advance online publication.

Apostolou, M., Frantzides, N., \& Pavlidou, A. (2014). Men competingmen watching: exploring watching pattern contingencies in sports. International Journal of Sports Communication, 7, 462-476.

Buss, D. M. (2003). The evolution of desire: strategies of human mating (2nd ed.). New York: Basic Books.

Cohen, J. (1988). Statistical power analysis for the behavioral sciences (2nd ed.). New York: Academic.

De Block, A., \& Dewitte, S. (2009). Darwinism and the cultural evolution of sports. Perspectives in Biology and Medicine, 52, 1-16.

Deaner, R. O., \& Smith, B. A. (2013). Sex differences in sports across 50 societies. Cross-Cultural Research, 47, 268-309.

Guttmann, A. (1986). Sports spectators. New York: Columbia University Press.

Guttmann, A. (2004). Sports: the first five millennia. Amherst: University of Massachusetts Press. 
Kyle, D. G. (2006). Sport and spectacle in the ancient world. New York: Wiley-Blackwell.

Lassek, W. D., \& Gaulin, S. J. C. (2009). Costs and benefits of fat-free muscle mass in men: relationship to mating success, dietary requirements, and native immunity. Evolution and Human Behavior, 30, 322-328.

Lombardo, M. P. (2012). On the evolution of sport. Evolutionary Psychology, 10, 1-28.

Miller, G. (2000). The mating mind. New York: Anchor Books.

Puts, D. A. (2010). Beauty and the beast: mechanisms of sexual selection in humans. Evolution and Human Behavior, 31, 157-175.

Seiler, S., DeKoning, J. J., \& Foster, C. (2007). The fall and rise of the gender difference in elite anaerobic performance 1952-2006. Medicine and Science in Sports and Exercise, 39, 534-540.
Tabachnick, B. G., \& Fidell, L. S. (2012). Using multivariate statistics (6th ed.). New York: Pearson.

Tooby, J., \& DeVore, I. (1987). The reconstruction of hominid behavioural evolution through strategic modelling. In W. G. Kinzey (Ed.), The evolution of human behaviour: primate models (pp. 183-237). Albany: State University of New York Press.

Trivers, R. L. (1972). Parental investment and sexual selection. In B. Campbell (Ed.), Sexual selection and the descent of man: 18711971 (pp. 136-179). Chicago: Aldine.

Zahavi, A., \& Zahavi, A. (1997). The handicap principle: A missing piece of Darwin's puzzle. New York: Oxford University Press. 